\title{
Avaliação da deglutição e indicadores clínicos associados em crianças após correção cirúrgica de doença cardíaca congênita
}

\section{Evaluation of swallowing and associated clinical markers in infants after surgical repair of congenital heart disease}

\author{
Heloisa Regina Fernandes ${ }^{1}$ (D), Fernanda Chiarion Sassi ${ }^{2}$ (1), Claudia Regina Furquim de Andrade ${ }^{2}$ (D), \\ Debora Maria Befi Lopes²
}

\section{RESUMO}

Objetivo: Identificar os marcadores clínicos associados às alterações da deglutição em crianças após a correção da doença cardíaca congênita e correlacionar esses marcadores às categorias da escala Risk Adjustment for Congenital Heart Surgery 1 (RACHS-1). Métodos: Foi realizado um estudo retrospectivo observacional, utilizando a base de dados eletrônica do centro de pediatria para crianças admitidas em um hospital, em decorrência de doença cardíaca congênita. Foram coletados dados da avaliação de deglutição e a presença de disfagia foi classificada de acordo com a Dysphagia Management Staging Scale (DMMS). Foram incluídos os dados demográficos, os marcadores clínicos e o risco de mortalidade, de acordo com a RACHS-1. Resultados: A amostra final do estudo foi composta por 108 pacientes. Os achados mais relevantes foram: os sinais mais graves de disfagia estiveram associados ao aumento da permanência no hospital $(\mathrm{p}=0,005)$; ao maior número e tempo de intubações orotraqueais $(\mathrm{p}=0,022$ e 0,005 , respectivamente); ao maior tempo entre a admissão hospitalar e a avaliação da deglutição $(\mathrm{p}=0,003)$; ao maior tempo entre o procedimento cirúrgico e a avaliação da deglutição $(\mathrm{p}=0,043)$ e ao maior número de sessões fonoaudiológicas para remoção da via alternativa de alimentação e retorno seguro para via oral $(p<0,001)$. Não foram encontradas correlações entre o risco de mortalidade das crianças e os sinais alterados na avaliação da deglutição. Conclusão: Os resultados do estudo contribuem para o conhecimento atual de que crianças com doenças cardíacas que precisam de correção cirúrgica apresentam dificuldades alimentares e necessitam atendimento hospitalar prolongado. Não houve correlação entre as categorias da escala RACHS-1 e os sinais de alteração na avaliação clínica da deglutição.

Palavras-chave: Desordens da deglutição; Alimentação; Deglutição; Doença cardíaca congênita; Mortalidade

\begin{abstract}
Purpose: To identify clinical markers that are associated to swallowing alterations in infants after surgical repair of congenital heart disease (CHD) and to correlate these markers to the categories on the Risk Adjustment for Congenital Heart Disease 1 (RACHS-1) scale. Methods: Using the Pediatric Center Database System we conducted a retrospective, observational cohort study on children admitted to the pediatric hospital unit due to $\mathrm{CDH}$. We collected data on specific parameters of a clinical swallowing assessment (SA) and dysphagia was classified according to the Dysphagia Management Staging Scale. We also included demographic and clinical markers and patient's risk of mortality was determined by using the RACHS-1. Results: The final study sample consisted of 108 patients. Important findings were: the more severe signs of dysphagia are associated to an increased length of hospital stay ( $\mathrm{p}=0.005)$; an increased number and duration of orotracheal intubation ( $\mathrm{p}=0.022$ and 0.005 respectively); an increased time between hospital admission and $\mathrm{SA}(\mathrm{p}=0.003)$; an increased time between the surgical procedure and swallowing assessment (0.043); and an increased number of SLP sessions to remove alternate feeding methods and warrant safe oral feeding $(p<0.001)$. No correlations were observed between the infant's risk of mortality and the altered sings on the clinical swallowing assessment. Conclusion: The data from this study contributes to the current knowledge that children with heart condition requiring heart surgery in the first month of life have high risk of presenting feeding difficulties and will require prolonged hospital care. No correlation was observed between the categories on RACHS-1 and the altered signs on the clinical swallowing assessment.
\end{abstract}

Keywords: Swallowing disorders; Feeding; Swallowing; Congenital heart defect; Mortality

Trabalho realizado no Instituto do Coração, Divisão de Fonoaudiologia, Hospital das Clínicas, Faculdade de Medicina, Universidade de São Paulo - USP - São Paulo (SP), Brasil.

${ }^{1}$ Instituto do Coração, Divisão de Fonoaudiologia, Hospital das Clínicas, Faculdade de Medicina, Universidade de São Paulo - USP - São Paulo (SP), Brasil. ${ }^{2}$ Departamento de Fisioterapia, Fonoaudiologia e Terapia Ocupacional, Faculdade de Medicina, Universidade de São Paulo - USP - São Paulo (SP), Brasil. Conflito de interesses: Não.

Contribuição dos autores: HRF foi responsável pela coleta de dados, análise e interpretação dos resultados; FCS foi responsável pela organização e condução da análise estatística, interpretação dos resultados e elaboração do manuscrito; CRFA foi responsável pela análise dos dados, interpretação dos resultados, escrita e revisão da versão final do manuscrito; DMBL foi responsável pela pesquisa e o desenho do estudo, contribuiu para a análise dos dados e preparação do manuscrito. Financiamento: Nada a declarar.

Autor correspondente: Fernanda Chiarion Sassi. E-mail: fsassi@usp.br

Recebido: Junho 17, 2020; Aceito: Fevereiro 15, 2021 


\section{INTRODUÇÃO}

A doença cardíaca congênita (DCC), normalmente definida como uma malformação do coração ou dos vasos sanguíneos que se desenvolvem durante o período fetal, é a principal causa de morte em bebês menores de 1 ano de idade ${ }^{(1)}$. De acordo com a literatura, a incidência varia entre quatro e dez por 1000 nascidos vivos, o que corresponde a cerca de 1.35 milhões de recém-nascidos por ano ${ }^{(2)}$. Avanços no diagnóstico fetal, cuidado perinatal, anestesiologia cardiovascular e cirurgia em neonatos com DCC, nas últimas quatro décadas, aumentaram consideravelmente a expectativa de $v i^{(3)}$. No entanto, com o aumento da sobrevida global, surgiram muitas necessidades especiais, principalmente as relacionadas à alimentação (nutrição parenteral ou modificações nas estratégias de alimentação enteral) e suporte respiratório prolongado $^{(4)}$. A prevalência de distúrbios alimentares póscirúrgicos em bebês com DCC varia de $22 \%$ a $50 \%{ }^{(5)}$. O mau estado nutricional resultante de capacidades de alimentação inadequadas, frequentemente leva ao prolongamento da fase de recuperação, falha de crescimento e desnutrição ${ }^{(6)}$. De acordo com a literatura, os recém-nascidos com quadro cardíaco grave e com necessidade de cirurgia cardíaca nos primeiros meses de vida têm alto risco de apresentar dificuldades alimentares até os 2 anos de idade ${ }^{(7)}$.

As habilidades motoras precursoras da alimentação oral começam a se desenvolver no útero (ou seja, por volta das 16 semanas de gestação), de modo que, nos primeiros dias de vida, bebês saudáveis, nascidos a termo, são capazes de manter suas necessidades nutricionais completas por meio da ingestão oral $^{(8)}$. De acordo com a literatura, a alimentação oral segura depende da sincronia coordenada sucção-deglutição-respiração, que ocorre por volta das 34 semanas completas de gestação ${ }^{(8)}$. Bebês com doenças cardíacas lutam com a resistência física e coordenação necessárias para sugar, engolir e respirar durante a alimentação, a fim de equilibrar o consumo e gasto de calorias para fornecer nutrição adequada para o crescimento somático ${ }^{(1)}$. Além disso, a deglutição e a respiração têm a faringe como espaço anatômico comum. Problemas em ambos os processos ou a falta de sincronização entre eles, podem afetar a capacidade do bebê de proteger adequadamente suas vias aéreas durante a ingestão de fluidos/alimentos, tendo como consequência a aspiração de material estranho para as vias aéreas inferiores ${ }^{(9)}$.

As dificuldades na alimentação oral são frequentes em pacientes com doença arterial coronariana (DAC) e podem piorar após a cirurgia cardíaca. Conforme apontado na literatura, muitos bebês com DCC complexa não desenvolvem as habilidades para se alimentar por via oral e têm alta hospitalar com sonda de gastrostomia ou alimentação nasogástrica ${ }^{(10)}$. A etiologia das dificuldades de alimentação dessas crianças é ainda incerta. Vários estudos investigaram os fatores de risco e os preditores de má alimentação nessa população. De maneira geral, os estudos apontam a natureza da DCC, a duração da intubação, a duração dos métodos alternativos de alimentação, o refluxo gastroesofágico, a paralisia das pregas vocais e o tipo de procedimento cirúrgico como causadores de um impacto negativo na deglutição ${ }^{(4,10,11)}$.

O manejo de bebês com DCC requer uma abordagem multidisciplinar e, nesse sentido, os fonoaudiólogos são os responsáveis por avaliar e tratar os distúrbios da função motora oral e faríngea, gerenciar a reabilitação dos músculos faciais e cervicais, bem como aconselhar os médicos sobre as trocas de tubos e a reintrodução da ingestão alimentar oral ${ }^{(12)}$. A participação desses profissionais em equipes multiprofissionais visa prevenir e reduzir complicações decorrentes de alterações da função motora oral ${ }^{(1,12)}$, diminuindo o tempo de internação e as reinternações por complicações ${ }^{(1)}$.

Considerando que a morbidade entre as crianças com DCC está relacionada a problemas de alimentação, como a presença de disfagia, os objetivos deste estudo foram: identificar marcadores clínicos que estão associados às alterações da deglutição nessa população e correlacionar esses marcadores com as categorias da escala Risk Adjustment for Congenital Heart Surgery 1 (RACHS-1) ${ }^{(13)}$. A identificação de indicadores de prioridade para o atendimento fonoaudiológico é fundamental para aprimorar a avaliação fonoaudiológica e promover o reinício seguro da alimentação por via oral. A hipótese desta pesquisa é a de que crianças com cardiopatias congênitas mais graves apresentarão alterações de deglutição mais graves e levarão mais tempo para fazer a transição da alimentação por sonda para a via oral.

\section{MÉTODOS}

Estudo de coorte observacional retrospectivo, aprovado por comitê de ética em pesquisa, e realizado no Instituto do Coração - InCor, da Faculdade de Medicina da Universidade de São Paulo, (Processo CAPPesq n $\left.{ }^{\circ} 1.856 .892\right)$. Trata-se de hospital terciário com 535 leitos (crianças e adultos), que recebe pacientes cardiopatas de todo o país. Uma vez que o estudo foi aprovado como revisão retrospectiva de documentos, o consentimento da família não foi necessário.

\section{População de pacientes}

Casos consecutivos de crianças internadas em unidade de terapia intensiva pediátrica para recuperação após correção cirúrgica de DCC, entre setembro de 2014 e agosto de 2017 , foram identificados por meio do sistema de dados da pediatria. Os pacientes eram elegíveis se atendessem a todos os seguintes critérios: idade entre 1 e 12 meses; ausência de tubo de traqueostomia ou gastrostomia; ausência de déficit neurológicos; ausência de síndromes genéticas e anomalias craniofaciais; estabilidade clínica e estado de alerta adequado; submetidos à avaliação clínica da deglutição (AD) por fonoaudiólogo, no mínimo, 24 horas após a extubação.

\section{Características demográficas e marcadores clínicos}

Foram coletadas as seguintes variáveis para investigar as características demográficas e os marcadores clínicos associados à disfagia: idade na $\mathrm{AD}$ (em meses), gênero, diagnóstico da cardiopatia, tipo de cardiopatia (cianótica ou acianótica), tempo de circulação extracorpórea no último procedimento cirúrgico (em minutos), dias de hospitalização, número de intubações orotraqueais, duração da intubação orotraqueal (em horas), tempo entre admissão hospitalar e AD (em dias), tempo entre a extubação e a $\mathrm{AD}$ (em dias), tempo entre o procedimento cirúrgico e AD (em dias), número de sessões de estimulação fonoaudiológica para retirar a via alternativa de alimentação, desfecho do paciente (alta fonoaudiológica, alta hospitalar, transferência hospitalar, óbito).

\section{Risco de mortalidade do paciente}

$\mathrm{O}$ risco de mortalidade do paciente foi determinado pela RACHS-1 ${ }^{(13)}$, método baseado em consenso de ajuste de risco 
para mortalidade intra-hospitalar entre crianças menores de 18 anos, após a cirurgia para DCC. Os procedimentos cirúrgicos são classificados em seis categorias de risco, permitindo comparar a mortalidade intra-hospitalar de grupos de crianças operadas para DCC. Para o presente estudo, os pacientes foram retrospectivamente classificados em uma das categorias da RACHS-1, após a verificação de qual procedimento cirúrgico foi realizado para cada um deles. Para cirurgias realizadas com procedimentos combinados, foi atribuída a categoria referente ao procedimento de maior risco, conforme descrito por Jenkins et al. ${ }^{(14)}$.

\section{Avaliação da deglutição}

Antes de iniciar a avaliação da deglutição, foram coletadas informações sobre o uso de métodos alternativos de alimentação (presença de sonda nasogástrica, sonda orogástrica ou suspensão de dieta) e a condição respiratória (independência respiratória; cateter de oxigênio nasal ou oxigenoterapia por nebulização) de cada paciente. Para a avaliação da deglutição, cada criança foi submetida a uma avaliação clínica da alimentação/deglutição que seguiu um protocolo padrão (Pediatric Dysphagia Evaluation Protocol - PDEP) $)^{(15)}$, que é o protocolo atual utilizado rotineiramente na instituição onde foi realizado estudo, para avaliação da deglutição. A disfagia foi, então, classificada com base na Dysphagia Management Staging Scale (DMSS), proposta por Sheppard, Hochman e Baer - deglutição normal/ sem disfagia, disfagia leve, disfagia moderada-grave, disfagia profunda) $)^{(16)}$

O PDEP é um protocolo de avaliação brasileiro, destinado à avaliação da disfagia pediátrica e tem como objetivos principais identificar alterações na dinâmica da deglutição, considerando as etapas de desenvolvimento do sistema estomatognático; caracterizar os sinais clínicos sugestivos de penetração/aspiração laringotraqueal; avaliar o impacto da disfagia na alimentação; orientar o fonoaudiólogo na tomada de decisão clínica, em relação à alimentação e reabilitação. $\mathrm{O}$ protocolo inclui itens descritos anteriormente como eficazes na identificação de crianças com disfagia, ou outros distúrbios de deglutição, e itens relevantes para a alimentação: como a criança lida com secreções de saliva e a presença de salivação excessiva, observação da postura geral, padrões de posicionamento e movimento, padrões respiratórios, estado de excitação, incluindo capacidade de resposta, avaliação da estrutura e função oral e a alimentação oral, propriamente $\operatorname{dita}^{(17)}$. A avaliação da alimentação por via oral envolve a administração controlada de volumes líquidos ou purês/sólidos com ou sem assistência. Para tanto, é utilizada a forma usual de alimentação da criança (por exemplo, amamentação, alimentação com mamadeira, alimentação com colher) e são oferecidos alimentos que são consumidos rotineiramente pela criança. Conforme determinado pelos autores do protocolo, o teste pode ser repetido, se necessário, para confirmação dos resultados. Para a presente pesquisa, foram utilizados mamadeiras e bicos padrão disponíveis no hospital. A avaliação da alimentação iniciou com a oferta de $5 \mathrm{ml}$ de uma fórmula comercial por, pelo menos, cinco minutos.

Após a aplicação do $\operatorname{PDEP}^{(15)}$, a disfagia foi classificada com base na Dysphagia Management Staging Scale ${ }^{(16)}$ da seguinte forma: (1) normal - sem sinais ou sintomas de distúrbio de deglutição no preparo oral e estágios orofaríngeos da deglutição; (2) disfagia leve - presença de sinais clínicos inadequados de deglutição durante a avaliação da alimentação. O distúrbio da deglutição pode ser tratado com um único tipo de estratégia: utensílios adaptados, restrições alimentares e mudanças posturais; (3) disfagia moderada-grave - a deglutição não é segura, com alto risco de interrupções na fase faríngea da deglutição. São observados problemas nutricionais e de hidratação relacionados. A consistência dos alimentos precisa ser modificada ou métodos alternativos de alimentação são necessários; (4) disfagia profunda - o indivíduo não consegue engolir com segurança pela boca, há risco elevado de aspiração. Toda a nutrição e a hidratação são recebidas por meios não orais (por exemplo, sonda nasogástrica) e pode ser necessário aspiração de saliva.

Para avaliar a confiabilidade dos dados, todos os participantes foram avaliados por dois fonoaudiólogos, com mais de 7 anos de experiência clínica, e independentes. Os fonoaudiólogos que realizaram as avaliações da deglutição foram aprovados em testes específicos de treinamento. O coeficiente Kappa foi utilizado para verificar a concordância entre os examinadores. O resultado obtido indicou um alto nível de concordância $(>0,866)$ para todas as características clínicas observadas.

\section{Análise de dados}

A análise foi realizada usando o software SPSS para Windows, versão 25.0. Analisaram-se, exclusivamente, as variáveis do PDEP relacionadas aos sinais e sintomas de disfagia. Esses itens foram agrupados e a presença de alterações foi analisada de acordo com a classificação da disfagia. As variáveis do PDEP foram agrupadas nas seguintes categorias:

a) Alterações na fase oral da deglutição que não comprometem a nutrição/hidratação: selamento labial e pega do mamilo (amamentação); mamilo, canudo e pega do copo; alimentação com colher; movimentos da língua (purê de sólidos); etapa de preparação oral (sólidos); padrões de mastigação;

b) Alterações da fase oral da deglutição que comprometam a nutrição/hidratação: coordenação sucção-deglutiçãorespiração (amamentação); tempo de trânsito oral; resíduo na cavidade oral; tempo de alimentação; recusa alimentar;

c) Alterações da frequência cardíaca e respiratória;

d) Dessaturação de oxigênio;

e) Sinais de dificuldade respiratória - dilatação nasal; retrações intercostais e subcostais; agitação;

f) Alterações na fase faríngea da deglutição - ausculta cervical; tosse; asfixia; qualidade vocal.

Os dados foram analisados no software IBM SPSS, versão 25. Além da análise descritiva, as comparações entre grupos foram realizadas por meio do teste de Mann-Whitney (para dados quantitativos), ou do Qui-quadrado de Pearson (para dados categóricos). A análise para verificar a presença de correlação entre as variáveis foi realizada por meio do coeficiente de correlação de Spearman. O nível de significância adotado foi de $5 \%$ para todas as análises. 


\section{RESULTADOS}

Após a aplicação dos critérios de inclusão, a amostra final do estudo foi composta por 108 pacientes. Os diagnósticos cardíacos das crianças incluídas foram os seguintes: comunicação intraventricular $(\mathrm{n}=16)$, tetralogia de Fallot $(\mathrm{n}=15)$, dupla via de saída do ventrículo direito $(\mathrm{n}=14)$, atresia tricúspide/ pulmonar $(\mathrm{n}=13)$, defeito do septo atrioventricular parcial ou total $(n=6)$, transposição das grandes artérias $(n=6)$, entrada dupla do ventrículo direito ou esquerdo $(\mathrm{n}=5)$, síndrome hipoplásica do coração esquerdo $(\mathrm{n}=5)$, coarctação da aorta $(n=4)$, conexão venosa pulmonar anômala total $(n=4)$, interrupção do arco aórtico $(\mathrm{n}=4)$, estenose aórtica/mitral ou pulmonar $(\mathrm{n}=3)$, artéria coronária esquerda anômala da artéria pulmonar $(\mathrm{n}=3)$, conexão anômala parcial de veias pulmonares na veia cava superior $(\mathrm{n}=2)$, janela aortopulmonar $(\mathrm{n}=2)$, truncus arteriosus $(\mathrm{n}=2)$, transposição congênita corrigida das grandes artérias $(n=2)$, hipoplasia aórtica/mitral $(n=1)$, artéria pulmonar direita anômala da artéria pulmonar $(\mathrm{n}=1)$.

Os pacientes foram agrupados de acordo com a classificação da disfagia. Do total de crianças da amostra, 27 foram diagnosticadas com disfagia leve (correspondendo a $25,0 \%$ da amostra); 79 foram diagnosticadas com disfagia moderada a grave (correspondendo a $73,1 \%$ da amostra) e 2 foram diagnosticadas com disfagia profunda (correspondendo a 1,9\% da amostra). Para fins estatísticos, esses dois últimos grupos foram mesclados. Assim, o número total de participantes do grupo com diagnóstico de disfagia moderada a grave foi de 81 (75,0\% da amostra). Em relação à presença de lesão/paralisia das pregas vocais (informações obtidas nos prontuários), é importante observar que os pacientes com disfagia leve não apresentavam alterações nas pregas vocais. A alteração das pregas vocais, entretanto, foi diagnosticada em 34 pacientes classificados com disfagia moderada a grave.

A Tabela 1 apresenta as comparações entre os grupos para as características demográficas e marcadores clínicos.

Os resultados indicaram que os dados demográficos relacionados à idade e gênero não diferenciaram os grupos, o que também foi observado para os marcadores clínicos relacionados ao tipo de cardiopatia, tempo de circulação extracorpórea no último procedimento cirúrgico e tempo entre a extubação e a avaliação da deglutição. Diferenças entre os grupos, no entanto, foram observadas para todos os outros marcadores clínicos. Os pacientes classificados com disfagia moderada a grave apresentaram resultados piores, quando comparados aos pacientes com disfagia leve. Considerando os resultados dos desfechos dos pacientes, apenas $1 / 3$ dos pacientes com disfagia moderada-grave apresentou alta fonoaudiológica, indicando que a maioria dos pacientes desse grupo permaneceu com alteração alimentar.

A Tabela 2 apresenta as comparações entre os grupos para o risco de mortalidade dos pacientes de acordo com a RACHS-1.

Os resultados indicaram que os grupos diferiram apenas na comparação do número de participantes classificados nas categorias 2 e 5 da RACHS-1. Proporcionalmente, o grupo com disfagia leve apresentou maior número de participantes nessas categorias; já o grupo de pacientes com disfagia moderada a grave, foi distribuído entre as categorias de maior risco.

A Tabela 3 mostra a comparação entre os grupos dos parâmetros obtidos na avaliação da deglutição.

Os resultados indicaram que os parâmetros métodos alternativos de alimentação, alterações na fase oral da deglutição que não comprometam a nutrição/hidratação, dessaturação de oxigênio, aumento da frequência cardíaca e/ou respiratória, sinais de dificuldade respiratória, frequência e qualidade vocal alterada não diferenciaram os grupos. De maneira geral, as crianças

Tabela 1. Comparação entre grupos para características demográficas e marcadores clínicos

\begin{tabular}{|c|c|c|c|}
\hline & $\begin{array}{l}\text { Disfagia orofaríngea leve } \\
(\mathrm{n}=27)\end{array}$ & $\begin{array}{l}\text { Disfagia orofaríngea } \\
\text { moderada-grave }(n=81)\end{array}$ & valor de $p$ \\
\hline Idade em meses (média $\pm \mathrm{DP}$ ) & $4,6( \pm 3,3)$ & $4,1( \pm 2,8)$ & 0,519 \\
\hline Gênero & Masculino = $18(67 \%)$ & Masculino = $38(47 \%)$ & 0,075 \\
\hline \multirow[t]{2}{*}{ Tipo de cardiopatia } & Cianótica = 14 (52\%) & Cianótica = 54 (67\%) & 0,167 \\
\hline & Acianótica = $13(48 \%)$ & Acianótica = $27(33 \%)$ & \\
\hline $\begin{array}{l}\text { Tempo de circulação extracorpórea no último procedimento cirúrgico } \\
\text { em minutos }\end{array}$ & 111,9 & 115,8 & 0,698 \\
\hline Dias de hospitalização (média $\pm D P$ ) & $60,4( \pm 81,9)$ & $70,1( \pm 81,9)$ & $0,005^{*}$ \\
\hline Número de intubações orotraqueais (média $\pm D P$ ) & $1,2( \pm 0.8)$ & $1,6( \pm 0,9)$ & $0,022^{*}$ \\
\hline Tempo de intubação orotraqueal em horas (média $\pm D P$ ) & $276,3( \pm 365,0)$ & $531,1( \pm 541,3)$ & $0,005^{*}$ \\
\hline Tempo entre a internação hospitalar e a AD em dias (média $\pm D P$ ) & $29,7( \pm 31.4)$ & $44,3( \pm 34,5)$ & $0,003^{*}$ \\
\hline Tempo entre a extubação e a AD em dias (média $\pm D P$ ) & $8,2( \pm 8,3)$ & $9,3( \pm 9,1)$ & 0,499 \\
\hline Tempo entre o procedimento cirúrgico e a $\mathrm{AD}$ em dias (média $\pm \mathrm{DP}$ ) & $18,3( \pm 15,2)$ & $30,7( \pm 28,4)$ & $0,043^{*}$ \\
\hline \multirow{4}{*}{ Desfecho clínico } & Alta hospitalar = $9(33 \%)$ & Alta hospitalar $=45(56 \%)$ & \\
\hline & $\begin{array}{l}\text { Transferência hospitalar }=1 \\
(4 \%)\end{array}$ & $\begin{array}{c}\text { Transferência hospitalar }=5 \\
(6 \%)\end{array}$ & \\
\hline & $\begin{array}{l}\text { Desligamento } \\
\text { fonoaudiológico }=0\end{array}$ & $\begin{array}{l}\text { Desligamento } \\
\text { fonoaudiológico }=6(7 \%)\end{array}$ & \\
\hline & Óbito = $1(4 \%)$ & Óbito $=0$ & \\
\hline
\end{tabular}

*Diferença significativa de acordo com o teste de Mann-Whitney; **Diferença significativa de acordo com o teste Qui-quadrado de Pearson

Legenda: $\mathrm{n}$ = número de sujeitos; $\mathrm{DP}=$ desvio padrão; $\mathrm{AD}$ = avaliação da deglutição 
Tabela 2. Comparação entre grupos para o risco de mortalidade cirúrgica de acordo com a escala de risco ajustado para cirurgia em cardiopatias congênitas (Risk Adjustment for Congenital Heart Surgery 1)

\begin{tabular}{cccc}
\hline Categorias da RACHS-1 & Disfagia orofaríngea leve $(\mathbf{n = 2 7 )}$ & $\begin{array}{c}\text { Disfagia orofaríngea } \\
\text { moderada-grave }(\mathbf{n = 8 1 )}\end{array}$ & valor de $\mathbf{p}$ \\
\hline 1 & 0 & $2(2 \%)$ & 0,410 \\
2 & $13(48 \%)$ & $19(23 \%)$ & $0,015^{\star}$ \\
3 & $9(33 \%)$ & $40(49 \%)$ & 0,147 \\
4 & $1(4 \%)$ & $10(12 \%)$ & 0,199 \\
5 & $2(7 \%)$ & 0 & $0,013^{\star}$ \\
6 & $2(7 \%)$ & $20(25 \%)$ & 0,480 \\
\hline
\end{tabular}

*Diferença significativa de acordo com o teste Qui-quadrado de Pearson

Legenda: $\mathrm{n}$ = número de participantes; RACHS-1 = Risk Adjustment for Congenital Heart Surgery 1 (escala de risco ajustado para cirurgia em cardiopatias congênitas)

Tabela 3. Comparação entre grupos de acordo com a avaliação fonoaudiológica

\begin{tabular}{|c|c|c|c|}
\hline & $\begin{array}{l}\text { Disfagia orofaríngea leve } \\
\qquad(n=27)\end{array}$ & $\begin{array}{c}\text { Disfagia orofaríngea } \\
\text { moderada-grave }(n=81)\end{array}$ & valor de $p$ \\
\hline \multirow[t]{3}{*}{ Via de alimentação na avaliação } & SNE/SNG = $26(96 \%)$ & SNE/SNG = $69(85 \%)$ & 0,124 \\
\hline & $\mathrm{SOE} / \mathrm{SOG}=0$ & SOE/SOG = $10(12 \%)$ & 0,055 \\
\hline & Jejum $=1(4 \%)$ & Jejum $=2(3 \%)$ & 0,735 \\
\hline \multirow[t]{3}{*}{ Condição respiratória } & Ar ambiente = $11(41 \%)$ & Ar ambiente = $16(18 \%)$ & $0,029^{*}$ \\
\hline & $\begin{array}{l}\text { Cateter nasal de oxigênio = } \\
\qquad 12(44 \%)\end{array}$ & $\begin{array}{l}\text { Cateter nasal de oxigênio = } \\
\qquad 88(59 \%)\end{array}$ & 0,180 \\
\hline & $\begin{array}{l}\text { Nebulização de oxigênio }=4 \\
\qquad(15 \%)\end{array}$ & $\begin{array}{l}\text { Nebulização de oxigênio }=17 \\
(21 \%)\end{array}$ & 0,483 \\
\hline $\begin{array}{l}\text { Alterações de fase oral da deglutição que não comprometem } \\
\text { nutrição/hidratação }\end{array}$ & $21(78 \%)$ & $54(67 \%)$ & 0,446 \\
\hline $\begin{array}{l}\text { Alterações de fase oral da deglutição que não comprometem } \\
\text { nutrição/hidratação }\end{array}$ & $6(22 \%)$ & $56(69 \%)$ & $<0,001^{*}$ \\
\hline Alteração na ausculta cervical & 0 & $26(32 \%)$ & $0,001^{*}$ \\
\hline Dessaturação de oxigênio & 0 & $6(7 \%)$ & 0,143 \\
\hline Aumento de FC e/ou FR & 0 & $3(4 \%)$ & 0,310 \\
\hline Sinais de desconforto respiratório & $1(4 \%)$ & $11(14 \%)$ & 0,157 \\
\hline Tosse & 0 & $21(26 \%)$ & $0,003^{*}$ \\
\hline Engasgo & 0 & $16(18 \%)$ & $0,012^{*}$ \\
\hline Alteração na qualidade vocal & $2(7 \%)$ & $9(11 \%)$ & 0,606 \\
\hline
\end{tabular}

*Diferença significativa de acordo com o teste Qui-quadrado de Pearson

Legenda: $\mathrm{n}=$ número de sujeitos; $\mathrm{SNE}=$ sonda nasoenteral; $\mathrm{SNG}=$ sonda nasogástrica; $\mathrm{SOE}=$ sonda oroenteral; $\mathrm{SOG}=$ sonda orogástrica; $\mathrm{FC}=$ frequência cardíaca; FR = frequência respiratória

classificadas com disfagia moderada a grave apresentaram pior condição respiratória e parâmetros alterados na avaliação da deglutição, quando comparadas ao grupo com disfagia leve. A presença de alterações na fase oral da deglutição que comprometam a nutrição/hidratação, ausculta cervical alterada e presença de tosse e engasgo após a deglutição foram mais frequentes nesses pacientes.

As possíveis correlações entre as características demográficas e os marcadores clínicos e o risco de mortalidade (Tabela 4) e entre os parâmetros da avaliação da deglutição e o risco de mortalidade (Tabela 5) também foram analisadas. Para essas análises, considerou-se o seguinte: alto grau de correlação - faixa do coeficiente de correlação acima de 0,75 ; grau moderado de correlação - faixa do coeficiente de correlação entre 0,50 a 0,75 ; baixo grau de correlação - faixa do coeficiente de correlação entre 0,25 a 0,50 e nenhuma correlação, abaixo de 0,25 .

De acordo com a análise dos dados, observou-se: baixo grau de correlação entre o risco de mortalidade e a idade (ou seja, quanto maior o risco de mortalidade, mais jovem é a criança); o risco de mortalidade e o tipo de cardiopatia (ou seja, quanto maior o risco de mortalidade, maior a chance de a criança apresentar cardiopatia cianótica congênita); o risco de mortalidade e dias de hospitalização (ou seja, quanto maior o risco de mortalidade, maior o tempo de internação da criança); o risco de mortalidade e a duração da intubação orotraqueal (ou seja, quanto maior o risco de mortalidade, maior o tempo de intubação da criança); o risco de mortalidade e o tempo entre a admissão hospitalar e a avaliação da deglutição (ou seja, quanto maior o risco de mortalidade, maior o tempo para a criança apresentar estabilidade clínica para poder realizar a avaliação da deglutição); o risco de mortalidade e o tempo entre o procedimento cirúrgico e a avaliação da deglutição (ou seja, quanto maior o risco de mortalidade, maior o tempo para a recuperação cirúrgica e para atingir a estabilidade clínica, permitindo assim a avaliação da deglutição); o risco de mortalidade e o número de sessões de fonoaudiologia para remover a via alternativa de alimentação (ou seja, quanto maior o risco de mortalidade, maior o tempo para atingir uma alimentação por via oral segura). Não foram encontradas correlações significativas entre o risco de mortalidade e os parâmetros de avaliação da deglutição.

\section{DISCUSSÃO}

De maneira geral, os principais resultados do presente estudo para crianças com DCC foram: os sinais mais graves de disfagia estão associados a um maior tempo de internação; 
Tabela 4. Análise da correlação entre características demográficas, marcadores clínicos e o risco de mortalidade

\begin{tabular}{lcc}
\hline & \multicolumn{2}{c}{ Correlação com o escore RACHS-1 } \\
\cline { 2 - 3 } Idade em meses & r & $0,002^{*}$ \\
Gênero & $-0,294$ & 0,757 \\
Tipo de cardiopatia & $-0,030$ & $<0,001^{*}$ \\
Tempo de circulação extracorpórea & $-0,385$ & 0,289 \\
Dias de hospitalização & 0,103 & $<0,001^{*}$ \\
Número de intubações orotraqueais & 0,378 & 0,360 \\
Tempo de intubação orotraqueal & 0,089 & $0,019^{*}$ \\
Tempo entre internação hospitalar e AD & 0,225 & $0,003^{*}$ \\
Tempo entre procedimento cirúrgico e AD & 0,283 & $0,014^{*}$ \\
Número de sessões fonoaudiológicas até retirada de vias alternativas de alimentação & 0,235 & $0,007^{*}$ \\
Desfecho clínico & 0,278 & 0,440 \\
\hline
\end{tabular}

*Diferença significativa de acordo com o teste Qui-quadrado de Pearson

Legenda: $A D$ = avaliação da deglutição; RACHS-1 = Risk Adjustment for Congenital Heart Surgery 1 (escala de risco ajustado para cirurgia em cardiopatias congênitas); $r$ = coeficiente de correlação de Pearson

Tabela 5. Análise da correlação entre os parâmetros da avaliação de deglutição e o risco de mortalidade

\begin{tabular}{|c|c|c|}
\hline & \multicolumn{2}{|c|}{ Correlação com o escore RACHS-1 } \\
\hline & $\mathbf{r}$ & valor de $p$ \\
\hline Via alternativa de alimentação & 0,015 & 0,879 \\
\hline Condição respiratória & 0,161 & 0,097 \\
\hline Alterações em fase oral da deglutição que não comprometem nutrição/hidratação & 0,049 & 0,623 \\
\hline Alterações em fase oral da deglutição que comprometem nutrição/hidratação & 0,020 & 0,838 \\
\hline Alteração na ausculta cervical & 0,090 & 0,356 \\
\hline Dessaturação de oxigênio & 0,164 & 0,090 \\
\hline Aumento na FC e/ou FR & $-0,052$ & 0,593 \\
\hline Sinais de desconforto respiratório & 0,121 & 0,212 \\
\hline Tosse & 0,195 & $0,045^{\star}$ \\
\hline Engasgo & 0,114 & 0,241 \\
\hline Alteração na qualidade vocal & 0,007 & 0,948 \\
\hline
\end{tabular}

*Correlação significativa de acordo com o coeficiente de correlação de Spearman

Legenda: RACHS-1 = Risk Adjustment for Congenital Heart Surgery 1 (escala de risco ajustado para cirurgia em cardiopatias congênitas);

$\mathrm{FC}=$ frequência cardíaca; $\mathrm{FR}=$ frequência respiratória

ao aumento no número e na duração da intubação orotraqueal; ao aumento no tempo entre a admissão hospitalar e a avaliação da deglutição; ao aumento no tempo entre o procedimento cirúrgico e a avaliação da deglutição e a um maior número de sessões de reabilitação para remover a via alternativa de alimentação, garantindo a alimentação por via oral segura. Além disso, os resultados indicaram que os pacientes com disfagia moderada e grave apresentam maior probabilidade de receber alta hospitalar sem a resolução da disfagia. O estudo sugere, ainda, que não existe correlação entre o risco de mortalidade da criança cardiopata e a qualidade vocal alterada na avaliação clínica da deglutição.

Conforme esperado, os resultados confirmaram que a presença de complicações e o aumento no tempo de internação hospitalar estão associados às internações de crianças com DCC ${ }^{(18)}$ Estudos recentes têm chamado à atenção para os altos custos envolvidos na assistência hospitalar dessa população ${ }^{(3,5)}$ $\mathrm{O}$ uso da tecnologia moderna e os avanços na assistência médica aumentaram consideravelmente o tempo de vida de muitas crianças com $\mathrm{DCC}^{(3)}$. A utilização de novas opções de tratamento, no entanto, não ocorre sem custos. No caso dos países em desenvolvimento, é fácil concluir que as implicações financeiras na utilização dessas tecnologias têm um impacto maior sobre os sistemas de saúde, que, normalmente, trabalham com orçamento reduzido. Um fator que contribui para retardar a alta de pacientes que foram submetidos a procedimentos cirúrgicos cardíacos é a ingestão oral insuficiente para atender às demandas metabólicas e necessárias para que a criança atinja o peso adequado. A causa desse atraso se deve, principalmente, à presença de alterações nos mecanismos envolvidos no processo de deglutição(4). Na amostra deste estudo, aproximadamente $60 \%$ dos pacientes com disfagia leve foram capazes de fazer a transição da via alternativa de alimentação para alimentação para a via oral, contra $30 \%$ daqueles classificados com disfagia moderada a grave.

Os dados do presente estudo também contribuem para o conhecimento atual de que a intubação orotraqueal prolongada é um fator de risco associado à disfagia ${ }^{(5)}$. A deglutição é um processo complexo, que requer uma coordenação precisa entre mais de 25 músculos, envolvendo a contração de diversos músculos orofaciais, faríngeos, laríngeos, respiratórios e esofágicos, além de seis pares de nervos cranianos e dos lobos frontais ${ }^{(19)}$. Qualquer alteração nesse processo pode resultar em disfagia, aumentando as chances de aspiração pulmonar e retardando a administração adequada de nutrição por via oral ${ }^{(19)}$. Estudos indicam que a intubação orotraqueal pode afetar a função laríngea poucas horas após o posicionamento do tubo, e seus efeitos podem perdurar por muito tempo após a extubação ${ }^{(20)}$. 
As explicações para a associação entre intubação orotraqueal prolongada e a presença de disfagia estão relacionadas ao impacto da permanência do tubo na cavidade oral, faríngea e laríngea, uma vez que o reflexo da deglutição é desencadeado por quimo e/ou mecanorreceptores localizados na mucosa da faringe e laringe. Esses receptores podem sofrer alterações devido à presença prolongada do tubo na região orotraqueal ${ }^{(21)}$. $\mathrm{Na}$ amostra deste estudo, os pacientes com disfagia moderada a grave permaneceram quase o dobro de tempo intubados, quando comparados aos pacientes com disfagia leve.

Um dos desfechos mais comumente observados nas cirurgias realizadas em pacientes com DCC é a paralisia das pregas vocais, principalmente da prega vocal esquerda, o que pode ser atribuído à intubação orotraqueal prolongada ou ao tipo de reparo cirúrgico realizado ${ }^{(22)} \mathrm{Na}$ literatura, a incidência da paralisia de prega vocal nessa população pode variar entre $9 \%$ e $54 \%{ }^{(11)}$. No presente trabalho, observou-se que $40 \%$ dos pacientes com disfagia moderada a grave apresentavam paralisia de prega vocal, enquanto os pacientes com disfagia leve não apresentavam essa condição. Com base no aumento da incidência de lesão do nervo laríngeo, recorrente durante procedimentos cirúrgicos, e nas possíveis alterações no funcionamento do nervo craniano que inerva a região faríngea, é possível que os problemas alimentares apresentados por algumas crianças com DCC tenham etiologia no mecanismo faríngeo ${ }^{(11)}$

Da mesma forma, prejuízos na função laríngea, portanto, parecem aumentar o risco de aspiração em crianças com DCC ${ }^{(23)}$. A paralisia das pregas vocais pode levar ao aumento do risco de aspiração pulmonar devido à falha de adução das pregas vocais durante a deglutição ${ }^{(23)}$. Conforme descrito na literatura, a aspiração pulmonar é responsável pelo aumento dos episódios de pneumonia, em razão da necessidade de reintubação e do atraso na alta hospitalar. Além disso, a literatura também aponta que crianças com idade $\leq 1$ ano apresentam um risco significativamente maior de desenvolver pneumonia, em decorrência de falhas no acionamento dos mecanismos das defesas pulmonares ${ }^{(24)}$. Assim, a identificação precoce dos sinais e sintomas da disfagia pode diminuir a morbidade dessas crianças.

Mesmo que a aspiração broncopulmonar não seja percebida durante o exame clínico, diversos estudos já descreveram os sinais mais comuns indicativos da presença de disfagia orofaríngea, sendo eles: tosse, engasgo, pigarro, apneia, cianose, dessaturação de oxigênio, taquipneia, bradicardia, alterações da cor da pele, respiração úmida, voz molhada, chiado, estridor e congestão pulmonar $^{(16)}$. No presente estudo, as crianças com disfagia moderada a grave apresentaram ausculta cervical alterada, tosse e engasgo após a deglutição, enquanto as crianças com disfagia leve não apresentaram esses sinais ${ }^{(16)}$ Embora a tosse esteja presente em $26 \%$ dos pacientes classificados com disfagia moderada a grave, acredita-se que a presença desse sinal deve ser analisada com cautela. A tosse é o reflexo defensivo mais importante para a eliminação de secreções das vias aéreas. Os receptores da tosse são encontrados nas células superficiais que revestem o tecido do trato respiratório superior da laringe até os bronquíolos segmentares, podendo ser acionados tanto por estímulos químicos, quanto mecânicos ${ }^{(25)}$. De acordo com a literatura, as doenças cardíacas e pulmonares estão de tal modo intimamente relacionadas, que muitas vezes é difícil determinar qual é a causa primária da presença de tosse ${ }^{(26)}$. As complicações pulmonares na DCC são frequentes, podendo ser causadas pelo impacto estrutural nas vias aéreas, por mecanismos fisiopatológicos anormais que levam ao aumento do líquido no pulmão, e/ou pela presença de alguma doença pulmonar ${ }^{(27)}$. As infecções do trato respiratório também podem ser responsáveis pela hospitalização prolongada nas crianças com DCC, atrasando a realização da cirurgia cardíaca. Por esse motivo, acredita-se que, ao realizar uma avaliação da deglutição nessas crianças, a presença de tosse deve ser analisada à luz de outros sintomas que possam indicar a presença de um distúrbio de deglutição e descartar a presença de infecções do trato respiratório. Neste estudo, não se investigou a presença de pneumonia vigente nos pacientes incluídos

Por fim, cabe ressaltar que o presente estudo apresentou algumas limitações. Em primeiro lugar, os dados coletados foram derivados de crianças de uma única instituição e, portanto, podem refletir características locais dos pacientes e atributos únicos dos procedimentos adotados no centro de pediatria. Todavia, a amostra foi composta por pacientes consecutivos, que foram submetidos a procedimentos cirúrgicos e encaminhados para avaliação da deglutição. Dessa forma, acredita-se que os achados representam de forma adequada essa população de pacientes. Em segundo lugar, as crianças incluídas na amostra do estudo apresentaram diferentes subtipos de defeitos cardíacos congênitos e, portanto, os resultados não podem ser generalizados para um defeito específico. Embora tenha havido a tentativa de minimizar esse efeito, caracterizando a amostra de acordo com o risco de mortalidade, os resultados não foram capazes de encontrar correlações específicas entre a gravidade clínica dos pacientes e os parâmetros alterados na avaliação clínica da deglutição. As características precisas dos deficit de deglutição podem variar em diferentes diagnósticos cardíacos e, portanto, estudos futuros são necessários em grandes amostras, para realizar essa análise comparativa. Como terceiro ponto, deve-se considerar que a avaliação clínica das alterações da deglutição tem limitações evidentes e um exame de videofluoroscopia (VFS) seria necessário para confirmar a presença de aspiração broncopulmonar. No entanto, conforme discutido na literatura, as alterações da deglutição e a broncoaspiração também podem ser subestimadas pela VFS. Até o momento, não há dados disponíveis que indiquem a sensibilidade e especificidade da VFS para detectar a broncoaspiração em populações pediátricas ${ }^{(28)}$. Além disso, embora a VFS seja o padrão-ouro para estudar os mecanismos orais e faríngeos da disfagia e detectar a broncoaspiração, seria inviável realizar esse exame em todos os pacientes da amostra, considerando fatores como a idade, condição médica, custos etc. A avaliação clínica da deglutição pode ser usada para identificar pacientes com risco para disfagia. Finalmente, considerando as características deste estudo - de coorte, retrospectivo e observacional -, não foi possível recuperar as informações sobre possíveis infecções vigentes do trato respiratório durante a avaliação da deglutição, ponto que deve ser considerado em estudos futuros.

\section{CONCLUSÃO}

A presença de sinais mais graves de disfagia em crianças submetidas a procedimentos cirúrgicos para correção de cardiopatias congênitas está associada ao aumento do tempo de internação, aumento do número e da duração da intubação orotraqueal e ao aumento do tempo para remoção da via alternativa de alimentação (ou seja, essas crianças demoram mais para apresentar a estabilidade clínica necessária para realizar uma avaliação da deglutição e precisam de mais sessões de reabilitação da deglutição para apresentar uma alimentação 
por via oral segura). Além disso, os resultados não indicaram correlações significativas entre o risco de mortalidade (categorias na escala Risk Adjustment for Congenital Heart Surgery 1 RACHS-1) e os parâmetros da avaliação clínica da deglutição.

\section{REFERÊNCIAS}

1. Weston C, Husain SA, Curzon CL, Neish S, Kennedy GT, Bonagurio $\mathrm{K}$, et al. Improving outcomes for infants with single ventricle physiology through standardized feeding during the interstage. Nurs Res Pract. 2016;2016:9505629. http://dx.doi.org/10.1155/2016/9505629. PMid:27313883.

2. Pereira KR, Firpo C, Gasparin M, Teixeira AR, Dornelles S, Bacaltchuk $\mathrm{T}$, et al. Evaluation of swallowing in infants with congenital heart defect. Int Arch Otorhinolaryngol. 2015;19(1):55-60. PMid:25992152.

3. Dean PN, Hillman DG, McHugh KE, Gutgesell HP. Inpatient costs and charges for surgical treatment of hypoplastic left heart syndrome. Pediatrics. 2011;128(5):e1181-6. http://dx.doi.org/10.1542/peds.20103742. PMid:21987703.

4. Sables-Baus S, Kaufman J, Cook P, Cruz EM. Oral feeding outcomes in neonates with congenital cardiac disease undergoing cardiac surgery. Cardiol Young. 2012;22(1):42-8. http://dx.doi.org/10.1017/ S1047951111000850. PMid:21729496.

5. Maurer I, Latal B, Geissmann H, Knirsch W, Bauersfeld U, Balmer C. Prevalence and predictions of later feeding disorders in children who underwent neonatal cardiac surgery for congenital heart disease. Cardiol Young. 2011;21(3):303-9. http://dx.doi.org/10.1017/ S1047951110001976. PMid:21272426.

6. Kelleher DK, Laussen P, Teixeira-Pinto A, Duggan C. Growth and correlates of nutritional status among infants with hypoplastic left heart syndrome (HLHS) after stage 1 Norwood procedure. Nutrition. 2006;22(3):237-44. http://dx.doi.org/10.1016/j.nut.2005.06.008. PMid:16500550.

7. Medoff-Cooper B, Irving SY. Innovative strategies for feeding and nutrition in infants with congenitally malformed hearts. Cardiol Young. 2009;19(Supl 2):90-5. http://dx.doi.org/10.1017/S1047951109991673. PMid:19857355.

8. Pickler RH, Reyna BA. A descriptive study of bottle-feeding opportunities in preterm infants. Adv Neonatal Care. 2003;3(3):13946. PMid:12891838.

9. Dodrill P, Gosa MM. Pediatric dysphagia: physiology, assessment, and management. Ann Nutr Metab. 2015;66(Supl 5):24-31. http:// dx.doi.org/10.1159/000381372. PMid:26226994.

10. Indramohan G, Pedigo TP, Rostoker N, Cambare M, Grogan T, Federman MD. Identification of risk factors for poor feeding in infants with congenital heart disease and a novel approach to improve oral feeding. J Pediatr Nurs. 2017;35:149-54. http://dx.doi.org/10.1016/j. pedn.2017.01.009. PMid:28169036.

11. Tibbetts KM, Wu D, Hsu JV, Burton WB, Nassar M, Tan M. Etiology and long-term functional swallow outcomes in pediatric unilateral vocal fold immobility. Int J Pediatr Otorhinolaryngol. 2016;88:17983. http://dx.doi.org/10.1016/j.ijporl.2016.07.008. PMid:27497409.

12. ASHA: American-Speech-Language-Hearing Association. Model medical review guidelines for dysphagia services [Internet]. Rockville: ASHA; 2004 [cited 2016 Aug 3]. Available from: http://www.asha.org/ uploadedfiles/practice/reimbursement/medicare/DunCorpDysphHCE. pdf

13. Cavalcante CTMB, Souza NMG, Pinto VC Jr, Branco KMPC, Pompeu RG, Teles ACO, et al. Analysis of surgical mortality for congenital heart defects using RACHS-1 Risk Score in a Brazilian single center. Rev Bras Cir Cardiovasc. 2016;31(3):219-25. http:// dx.doi.org/10.5935/1678-9741.20160022. PMid:27737404.

14. Jenkins KJ, Gauvreau K, Newburger JW, Spray TL, Moller JH, Iezzoni LI. Consensus-based method for risk adjustment for surgery for congenital heart disease. J Thorac Cardiovasc Surg. 2002;123(1):1108. http://dx.doi.org/10.1067/mtc.2002.119064. PMid:11782764.

15. Sassi FC, Bühler KCB, Juste FS, Almeida FCF, Befi-Lopes DM, Andrade CRF. Dysphagia and associated clinical markers in neurologically intact children with respiratory disease. Pediatr Pulmonol. 2018;53(4):51725. http://dx.doi.org/10.1002/ppul.23955. PMid:29393599.

16. Sheppard JJ, Hochman R, Baer C. The dysphagia disorder survey: validation of an assessment for swallowing and feeding function in developmental disability. Res Dev Disabil. 2014;35(5):929-42. http:// dx.doi.org/10.1016/j.ridd.2014.02.017. PMid:24637033.

17. Weir K, McMahon S, Barry L, Masters IB, Chang AB. Clinical signs and symptoms of oropharyngeal aspiration and dysphagia in children. Eur Respir J. 2009;33(3):604-11. http://dx.doi.org/10.1183/09031936.00090308. PMid:19010985.

18. Pasquali SK, Jacobs JP, Bove EL, Gaynor JW, He X, Gaies MG, et al. Quality-cost relationship in congenital heart surgery. Ann Thorac Surg. 2015;100(4):1416-21. http://dx.doi.org/10.1016/j.athoracsur.2015.04.139. PMid:26184555.

19. Weckmueller J, Easterling C, Arvedson J. Preliminary temporal measurement analysis of normal oropharyngeal swallowing in infants and young children. Dysphagia. 2011;26(2):135-43. http://dx.doi. org/10.1007/s00455-010-9283-3. PMid:20532920.

20. Skoretz SA, Yau TM, Ivanov J, Granton JT, Martino R. Dysphagia and associated risk factors following extubation in cardiovascular surgical patients. Dysphagia. 2014;29(6):647-54. http://dx.doi.org/10.1007/ s00455-014-9555-4. PMid:25119447.

21. Larminat V, Montravers P, Dureuil B, Desmonts JM. Alteration in swallowing reflex after extubation in intensive care unit patients. Crit Care Med. 1995;23(3):486-90. http://dx.doi.org/10.1097/00003246199503000-00012. PMid:7874899.

22. Chen HC, Jen YM, Wang CH, Lee JC, Lin YS. Etiology of vocal cord paralysis. ORL J Otorhinolaryngol Relat Spec. 2007;69:167-71. http:// dx.doi.org/10.1159/000099226.

23. Benjamin JR, Smith PB, Cotten CM, Jaggers J, Goldstein RF, Malcolm WF. Long-term morbidities associated with vocal card paralysis after surgical closure of a patent ductus arteriosus in extremely low birth weight infants. J Perinatol. 2010;30(6):408-13. http://dx.doi. org/10.1038/jp.2009.124. PMid:19759545.

24. Taniguchi MH, Moyer RS. Assessment of risk factors for pneumonia in dysphagic children: significance of videofluoroscopic swallowing evaluation. Dev Med Child Neurol. 1994;36(6):495-502. http://dx.doi. org/10.1111/j.1469-8749.1994.tb11879.x. PMid:8005360.

25. Praprotnik M, Zupanic M, Lozej T, Krivec U. Cough in children. Zdrav Vestn. 2017;86:212-25.

26. Moss AJ, McDonald LV. Cardiac disease in the wheezing child. Chest. 1977;71(2):187-92. http://dx.doi.org/10.1378/chest.71.2.187. PMid:832490.

27. Healy F, Hanna BD, Zinman R. Pulmonary complications of congenital heart disease. Paediatr Respir Rev. 2012;13(1):10-5. http://dx.doi org/10.1016/j.prrv.2011.01.007. PMid:22208788.

28. Maffey A, Moviglia T, Mirabello C, Blumenthal L, Gentile L, Niremberg $\mathrm{M}$, et al. Swallowing and respiratory distress in hospitalized patients with bronchiolitis. Dysphagia. 2013;28(4):582-7. http://dx.doi. org/10.1007/s00455-013-9470-0. PMid:23689810. 